\title{
Electron beam computed tomography for the detection of left atrial thrombi in patients with atrial fibrillation
}

\author{
S Achenbach, D Sacher, D Ropers, K Pohle, U Nixdorff, U Hoffmann, G Muschiol, F A Flachskampf, \\ W G Daniel
}

A pproximately $13-15 \%$ of patients with atrial fibrillation have atrial thrombi, usually in the left atrial appendage (LAA). Transoesophageal echocardiography (TOE), frequently used before cardioversion, constitutes the routine clinical tool to detect atrial thrombi. ${ }^{1}$ TOE is a safe test, ${ }^{2}$ but may be considered not completely non-invasive, may require sedation, and relies on an experienced operator. The development of alternative imaging modalities to rule out atrial thrombi thus bears potential interest. We therefore evaluated the ability of electron beam computed tomography (EBCT), a computed tomography (CT) technique with high temporal resolution, to visualise atrial thrombi in patients with atrial fibrillation.

\section{METHODS}

We enrolled 52 consecutive patients (33 men, 19 women, mean (SD) age 66 (10) years, mean heart rate: 91 (24) minute) with atrial fibrillation of at least 48 hours duration, scheduled for direct current cardioversion. Patients with contraindications to iodinated contrast agent, post-prosthetic mitral valve replacement, in an unstable haemodynamic condition, or unable to give consent were not enrolled. The study protocol was approved by the institutional review board.

EBCT was performed before TOE using a C-150 XP EBCT scanner (GE/Imatron, South San Francisco, California, USA). Fifty cross sectional images of the left atrium were acquired in inspiration ( $1.5 \mathrm{~mm}$ slice thickness, $1.0 \mathrm{~mm}$ table increment, acquisition time $100 \mathrm{~ms}$ per image). Image acquisition was prospectively triggered to the ECG with one image being acquired $250 \mathrm{~ms}$ after the peak of each $\mathrm{R}$ wave. After determination of transit time with a $10 \mathrm{ml}$ bolus, $140 \mathrm{ml}$ contrast agent $(4 \mathrm{ml} / \mathrm{s})$ were injected intravenously. Two observers blinded to TOE results independently evaluated the EBCT datasets concerning presence of a thrombus (nonenhancing mass) in the left atrium. In case of disagreement, consensus was achieved in a final joint reading.

TOE was performed using a multiplane $5 \mathrm{MHz}$ transducer, stored on video tape, and evaluated for the presence of left atrial thrombus and spontaneous echo contrast (smoke) by two independent, experienced observers blinded to EBCT results. In case of disagreement, consensus was achieved in a final joint reading.

\section{RESULTS}

TOE demonstrated thrombi within the LAA in seven patients (13\%); thrombus was ruled out in 45 . In EBCT, all seven patients with atrial thrombi were correctly detected (fig 1). In 39 out of 45 patients without left atrial thrombus in TOE, EBCT correctly ruled out the presence of thrombus (sensitivity $100 \%$, specificity $87 \%$, positive predictive value $54 \%$, negative predictive value $100 \%$ ). In six of the 45 patients, inhomogeneous or reduced contrast enhancement in the LAA did not rule out the presence of a thrombus in EBCT. In five of these patients, spontaneous echo contrast (smoke) within the LAA was noted in TOE and the mean LAA flow velocity $(25(10) \mathrm{cm} / \mathrm{s})$ was significantly lower than in patients with a true negative EBCT finding $(43 \quad(18) \mathrm{cm} / \mathrm{s}, \mathrm{p}=0.04)$. Agreement between the two observers was achieved in 47 cases $(90 \%)$ in TOE and in 48 cases (92\%) in EBCT (Cohen's $\kappa$ 0.61 and 0.82 ).

\section{DISCUSSION}

We evaluated the ability of contrast enhanced EBCT to detect and rule out LAA thrombi in patients with atrial fibrillation. In a series of 52 patients scheduled for direct current cardioversion, EBCT correctly detected all seven patients in which TOE had shown a thrombus, while the presence of thrombus could be correctly ruled out in 39 of 45 patients. The lower LAA maximum emptying or filling flow velocity and high occurrence of spontaneous echo contrast in patients with false positive EBCT findings indicate that blood stasis and lack of exchange with the left atrial blood pool may have precluded sufficient contrast enhancement in the LAA. Some previous publications have reported on the use of CT techniques for the detection of left atrial thrombi. ${ }^{3-5}$ None of these studies were validated against TOE.

Limitations of this study include a small sample size with only seven patients in the group having a thrombus present. Outcome data were not obtained. Evaluation of TOE, used as the gold standard to validate EBCT, is subjective and disagreement between the observers occurred. The EBCT imaging protocol may not have been optimal for using $1.5 \mathrm{~mm}$ slice collimation (which has a high image noise) and lack of a second, post-contrast scan to compensate for filling defects caused by poor LAA function. ${ }^{3}$ We did not measure thrombus size which may be of importance for follow up investigations.

CT techniques, which require contrast agent and are associated with radiation exposure, are not likely to replace TOE as the diagnostic tool of choice to detect LAA thrombi in atrial fibrillation. Neither would they generally provide better cost effectiveness or risk/benefit ratio. However, TOE may not be feasible in certain situations and fast CT imaging may develop into a reliable alternative. Furthermore, the presence of LAA thrombus may be detected as an incidental finding in CT studies performed for another reason. Currently, EBCT provides the highest temporal resolution for cardiac CT, but given its rapid technical development, multi-slice CT (which, at the expense of higher radiation, provides higher spatial resolution, better signal to noise ratio and a shorter overall scan time, thus requiring less contrast agent) may soon constitute a more widely available alternative method. Magnetic resonance imaging would constitute another possible approach, but the necessity to average data acquired

Abbreviations: $E B C T$, electron beam computed tomography; LAA, left atrial appendage; TOE, transoesophageal echocardiography 

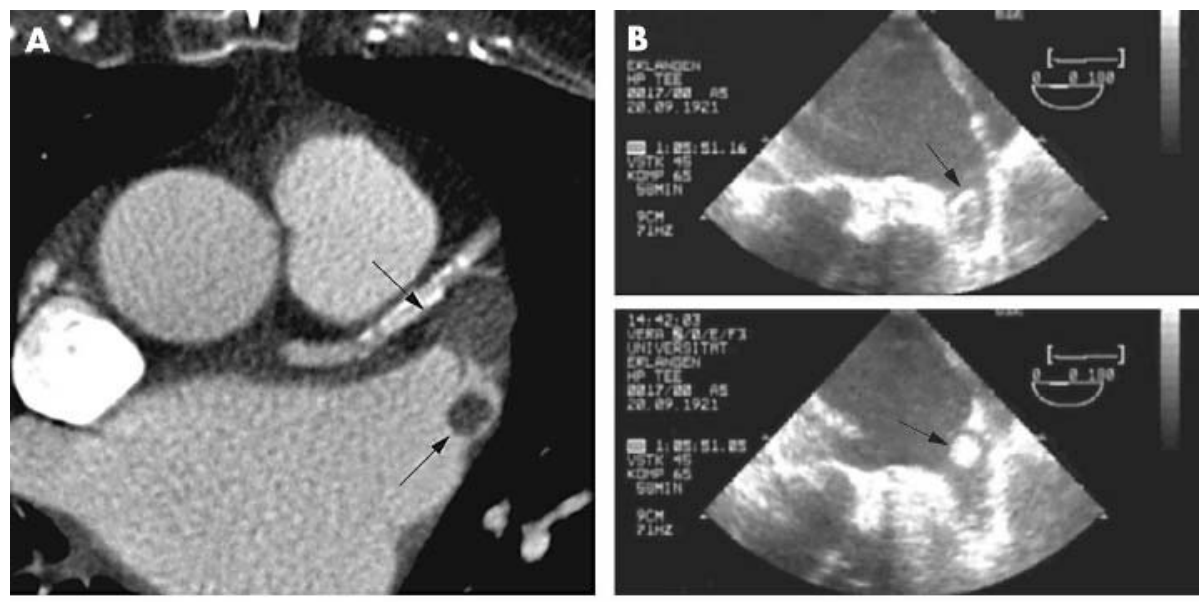

Figure 1 Visualisation of thrombi within the left atrium and LAA by EBCT. (A) EBCT shows lack of contrast enhancement in the apex of the LAA (large arrow) and a smaller filling defect in the region of the orifice of the atrial appendage (small arrow). (B) Two frames of the TOE investigation showing the two thrombi (arrows). (C) Normal visualisation and complete

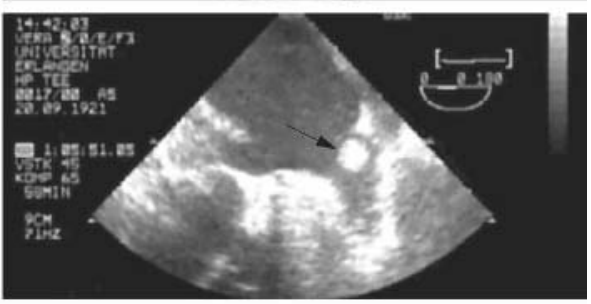
contrast enhancement of the LAA (arrow) in EBCT. (D) False positive finding by EBCT. Incomplete contrast enhancement of the LAA in EBCT could not rule out the presence of a thrombus (arrow). In TOE, no thrombus but spontaneous echo contrast was documented.
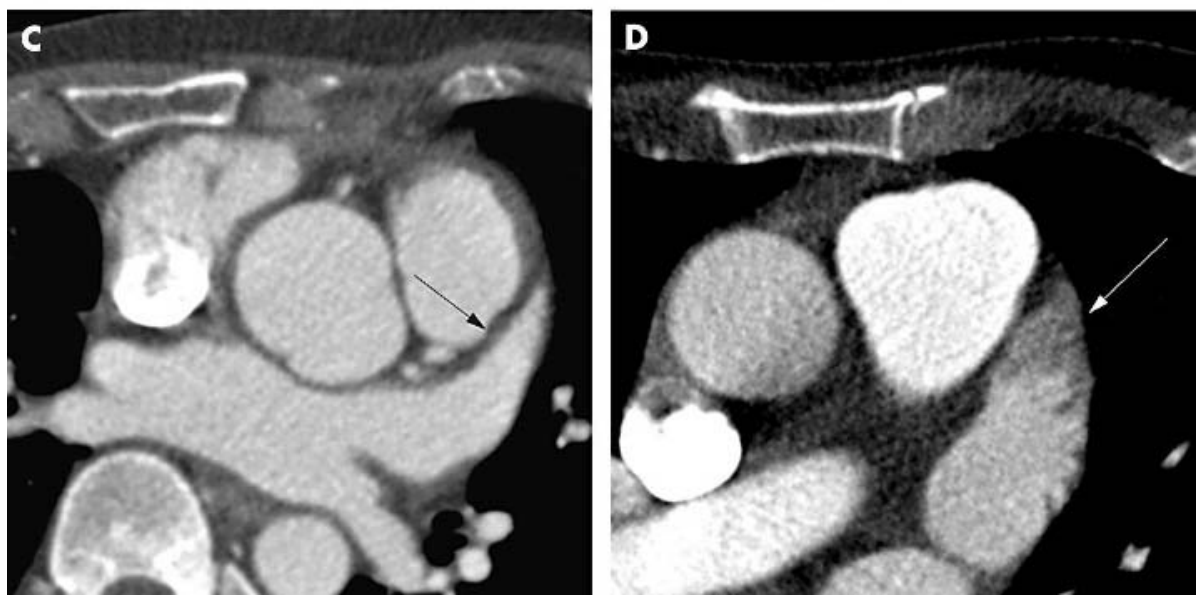

over several consecutive heart beats is a severe limitation given the irregular cycle length in atrial fibrillation.

\section{ACKNOWLEDGEMENTS}

This study was supported by the Staedtler-Foundation, Nürnberg, Germany. Dr Achenbach was supported by the Deutsche Forschungsgemeinschaft, Bonn, Germany. Dr Hoffmann was supported by the New York Cardiac Center.

\section{Authors' affiliations}

S Achenbach*, D Sacher, D Ropers, K Pohle, U Nixdorff, G Muschiol, F A Flachskampf, W G Daniel, Department of Internal Medicine II (Cardiology), University of Erlangen-Nürnberg, Germany

U Hoffmann, Cardiology Division, Massachusetts General Hospital, Boston, Massachusetts, USA

*Also at the Department of Radiology, Massachusetts General Hospital, Boston and the Cardiology Division, Massachusetts General Hospital, Boston, Massachusetts, USA
Correspondence to: Dr Stephan Achenbach, Department of Internal Medicine II, University of Erlangen, Ulmenweg 18, 91054 Erlangen, Germany; stephan.achenbach@med2.med.uni-erlangen.de

Accepted 16 February 2004

\section{REFERENCES}

1 Klein AL, Murray D, Grimm RA. Role of transesophageal echocardiographyguided cardioversion of patients with atrial fibrillation. J Am Coll Cardiol 2001;37:691-704.

2 Daniel WG, Erbel R, Kasper W, et al. Safety of transesophageal echocardiography. A multicenter survey of 10,419 examinations. Circulation $1991: 83: 817-22$

3 Nakanishi T, Hamada S, Takamiya M, et al. A pitfall in ultrafast CT scanning for the detection of left atrial thrombi. J Comput Assist Tomogr 1993; 17:42-5

4 Love BB, Struck LK, Stanford W, et al. Comparison of two-dimensional echocardiography and ultrafast cardiac computed tomography for evaluating intracardiac thrombi in cerebral ischemia. Stroke 1990;21:1033-8.

5 Tomoda H, Hoshiai M, Tagawa R, et al. Evaluation of left atrial thrombus with computed tomography. Am Heart J 1980;100:306-10. 